Research Article

\title{
Boundary Criteria for the Stability of Delay Differential-Algebraic Equations
}

\author{
Leping Sun and Yuhao Cong \\ College of Mathematics and Sciences, Shanghai Normal University, Shanghai 200234, China \\ Correspondence should be addressed to Leping Sun; sunlp611@126.com
}

Received 19 October 2014; Revised 17 April 2015; Accepted 24 April 2015

Academic Editor: Valery Y. Glizer

Copyright (C) 2015 L. Sun and Y. Cong. This is an open access article distributed under the Creative Commons Attribution License, which permits unrestricted use, distribution, and reproduction in any medium, provided the original work is properly cited.

\begin{abstract}
This paper is concerned with the asymptotic stability of delay differential-algebraic equations. Two stability criteria described by evaluating a corresponding harmonic analytical function on the boundary of a certain region are presented. Stability regions are also presented so as to show the method geometrically. Our results are not reported.
\end{abstract}

\section{Introduction}

Functional differential equations have a wide range of applications in science and engineering. Perhaps the most natural type of functional differential equation is a "delay differential equation," that is, differential equations with dependence on the past state. One type of past dependence is that it is carried out through the state variable but not through its derivative. Then the equation can be expressed as delay differential equation (DDE). Delay differential-algebraic equations (DDAEs), which have both delay and algebraic constraints, arise frequently in circuit simulation and power system, due to, for example, interconnects for computer chips and transmission lines, and in chemical process simulation when modeling pipe flows.

The criteria for the stability of DDAE can be classified into two categories according to their dependence upon the size of delays. The criteria that do not include information on delays are referred to as the delay independent criteria, while those carrying the information on delays are called the delay dependent criteria.

In this paper we are concerned with the asymptotic stability of a system of DDAEs:

$$
A x^{\prime}(t)+B x(t)+C x(t-\tau)=0,
$$

where $A, B, C \in \mathbb{C}^{d \times d}$ are constant real matrices, $A$ is singular, and $\tau>0$ stands for a constant delay. We will study delay independent criteria as well as delay dependent criteria for the above system. Our stability criteria only require the evaluation of a real function on the boundary of a certain region in the complex plane. The region is given as the intersection of a rectangle and a circle both specified with the system.

We will first introduce zeros of analytical function in a bounded region and the logarithmic norm of a matrix. Then the stability criteria of DDAEs are presented.

\section{Preliminary}

As a preliminary, we will introduce theorems of complexvariable functions. Let $W$ denote a bounded region of the complex plane. $\partial W$ and $\bar{W}$ represent the boundary and the closure of $W$, respectively. That is, $\bar{W}=\partial W \cup W$ :

$$
f(s)=f(x, y)=u(x, y)+i v(x, y)
$$

is an arbitrary analytical function for $s \in \bar{W}$. Here $i^{2}=-1$, $s=x+i y, u(x, y)=\operatorname{Re} f(s)$, and $v(x, y)=\operatorname{Im} f(s)$. The following two theorems give sufficient conditions for nonexistence of zeros of $f(s) \in \bar{W}$. The two theorems only require the evaluation on the boundary $\partial W$ of harmonic analytical functions each corresponding to $f$. Hence, they are called boundary criteria.

Theorem 1 (see [1]). If for any $(x, y) \in \partial W$, the real part $u(x, y)$ in (2) does not vanish, then $f(x, y) \neq 0$ for any $(x, y) \in$ $W$. 
Theorem 2 (see [1]). Assume that, for any $(x, y) \in \partial W$, there exists real constant $\lambda$ satisfying $u(x, y)+\lambda v(x, y) \neq 0$. Then $f(s)=u(x, y)+i(x, y) \neq 0$, for any $(x, y) \in \bar{W}$.

Theorem 2 is an extension of Theorem 1. The following four lemmas are important to our results.

Lemma 3 (see [2]). Let $A \in \mathbb{C}^{d \times d}$ and $B \in \mathbb{R}^{d \times d}$. If the inequality $|A| \leq B$ holds, then the inequality $\rho(A) \leq$ $\rho(B)$ is valid. Here the order relation of matrices of the same dimensions should be interpreted componentwise. $|A|$ stands for the matrix whose component is replaced by the modulus of the corresponding component of $A$, and $\rho(A)$ means the spectral radius of $A$.

For a complex matrix $W$, let $\mu(W)$ be the logarithmic norm of $W$ :

$$
\mu(W)=\lim _{\Delta \rightarrow 0^{+}} \frac{\|I+\Delta W\|-1}{\Delta} .
$$

$\mu(W)$ depends on the chosen matrix norm. Let $\|W\|$ denote the matrix norm of $W$ subordinate to a certain vector norm. In order to specify the norm, the notation $\|\cdot\|_{p}$ is used. And the notation $\mu_{p}(\cdot)$ is also adopted to denote the logarithmic norm associated with $\|\cdot\|_{p}$.

Lemma 4 (see [2]). For each eigenvalue of a matrix $W \in$ $\mathbb{C}^{d \times d}$, the inequality

$$
-\mu_{p}(-W) \leq \operatorname{Re} \lambda_{i}(W) \leq \mu_{p}(W)
$$

holds.

Lemma 5 (see [3]). Let $U, V$ be $n \times k$ rectangular matrices with $k \leq n$, and let $A$ be an $n \times n$ matrix; then

$$
T=I+V^{T} A^{-1} U
$$

is nonsingular if and only if $A+U V^{T}$ is nonsingular. In this case, one has

$$
\left(A+U V^{T}\right)^{-1}=A^{-1}-A^{-1} U T^{-1} V^{T} A^{-1} .
$$

Lemma 6 (see [3]). Let $\|\cdot\|$ be a norm defined on $\mathbb{C}^{n \times n}$ with $\|I\|=1$ and $A \in \mathbb{C}^{n \times n}$ satisfy $\|A\|<1$. Then $I-A$ is nonsingular and satisfies

$$
\left\|(I-A)^{-1}\right\| \leq \frac{1}{1-\|A\|} .
$$

\section{Delay Independent Stability of DDAEs}

Now we deal with the asymptotic stability of DDAEs,

$$
A x^{\prime}(t)+B x(t)+C x(t-\tau)=0
$$

where $A, B, C \in \mathbb{R}^{d \times d}$ are constant real matrices, $A$ is singular, and $\tau>0$ stands for a constant delay. According to [4], the system is solvable if and only if the matrix pencil $\lambda A+B$ is regular, that is, not identically singular for any $\lambda$. Thus, for the system to be asymptotically stable, $B$ is required to be nonsingular since; otherwise, $\lambda=0$ will be a singular value of the matrix pencil.

For the stability of system (8), we investigate its characteristic equation:

$$
P(\lambda)=\operatorname{det}\left[\lambda A+B+C e^{-\lambda \tau}\right]=0 .
$$

When $\lambda \neq 0$ and letting $s=1 / \lambda$, then the above characteristic equation may be written as

$$
\operatorname{det}\left[\frac{1}{s} A+B+C e^{-\tau / s}\right]=0
$$

If $\left\|B^{-1}\right\| \cdot\|C\|<1$, matrix $\left(B+C e^{-\tau / s}\right)$ is nonsingular, so (10) may be written as

$$
\operatorname{det}\left[s I+A\left(B+C e^{-\tau / s}\right)^{-1}\right]=0
$$

and (11) also may be written as

$$
\operatorname{det}\left[s I+A\left(B+C e^{-\tau / s}\right)^{-1}\right]=U(x, y)+i V(x, y)
$$

where $s=x+i y$. By the above assumption, $\operatorname{Re}(s)<0 \Leftrightarrow$ $\operatorname{Re} \lambda<0$ is valid.

The following lemma is well known.

Lemma 7 (see [5]). If the real parts of all the characteristic roots of (11) are less than zero, then system (8) is asymptotically stable; that is, solution $x(t)$ of (8) satisfies $x(t) \rightarrow 0$ as $t \rightarrow$ $\infty$. (8).

The following is a sufficient condition for the stability of

Lemma 8. Let $\left\|B^{-1}\right\| \cdot\|C\|<1$. If the condition

$$
\mu\left(-A B^{-1}\right)+\frac{\|A\|\left\|B^{-1}\right\|^{2}\|C\|}{1-\left\|B^{-1}\right\|\|C\|}<0
$$

holds, system (8) is asymptotically stable.

Proof. Assume that the condition of the lemma is satisfied and that system (8) is unstable. There is a characteristic root of (11) with positive real part. Note that the characteristic root is an eigenvalue of the matrix $-A\left(B+C e^{-\tau / s}\right)^{-1}$. By Lemma 5 , we have the following inequalities:

$$
0 \leq \operatorname{Re}(s) \leq \mu\left[-A\left(B+C e^{-\tau / s}\right)^{-1}\right]
$$


Applying the properties of the logarithmic norm and Lemmas 5 and 6 , we have

$$
\begin{aligned}
\mu & \left.-A\left(B+C e^{-\tau / s}\right)^{-1}\right] \\
= & \mu\left[-A\left(B^{-1}-B^{-1} C\left(I+B^{-1} C e^{-\tau / s}\right)^{-1} e^{-\tau / s} B^{-1}\right)\right] \\
= & \mu\left[-A B^{-1}+A B^{-1} C\left(I+B^{-1} C e^{-\tau / s}\right)^{-1} e^{-\tau / s} B^{-1}\right] \\
\leq & \mu\left(-A B^{-1}\right) \\
& +\left\|A B^{-1} C\left(I+B^{-1} C e^{-\tau / s}\right)^{-1} e^{-\tau / s} B^{-1}\right\| \\
\leq & \mu\left(-A B^{-1}\right) \\
& +\|A\|\left\|B^{-1}\right\|^{2}\|C\|\left\|\left(I+B^{-1} C e^{-\tau / s}\right)^{-1}\right\| \\
\leq & \mu\left(-A B^{-1}\right)+\frac{\|A\|\left\|B^{-1}\right\|^{2}\|C\|}{1-\left\|B^{-1}\right\|\|C\|} .
\end{aligned}
$$

This however contradicts the condition; hence, the proof is completed.

From the above lemma, if $\mu\left(-A B^{-1}\right)+\|A\|\left\|B^{-1}\right\|^{2}\|C\| /(1-$ $\left.\left\|B^{-1}\right\|\|C\|\right)<0$ is not satisfied, system (8) may be stable or unstable. By Lemma 5, we have the following inequalities:

$$
\begin{gathered}
-\mu\left[A\left(B+C e^{-\tau / s}\right)^{-1}\right] \leq \operatorname{Re} s \\
\leq \mu\left[-A\left(B+C e^{-\tau / s}\right)^{-1}\right] .
\end{gathered}
$$

A derivation similar to that of Lemma 8 leads to

$$
\begin{gathered}
-\mu\left(A B^{-1}\right)-\frac{\|A\|\left\|B^{-1}\right\|^{2}\|C\|}{1-\left\|B^{-1}\right\|\|C\|} \leq \operatorname{Re} s \\
\leq \mu\left(-A B^{-1}\right)+\frac{\|A\|\left\|B^{-1}\right\|^{2}\|C\|}{1-\left\|B^{-1}\right\|\|C\|} .
\end{gathered}
$$

So according to Lemma 8 , if $\mu\left(-A B^{-1}\right)+\|A\|\left\|B^{-1}\right\|^{2}\|C\| /(1-$ $\left.\left\|B^{-1}\right\|\|C\|\right)<0$ is not valid, there are two situations when the condition of Lemma 8 fails. The following theorem gives two regions from two situations including all the roots of (11) with nonnegative real parts.

Theorem 9. Let $\left\|B^{-1}\right\| \cdot\|C\|<1$. Suppose that there exists a root of (11) whose real part is nonnegative.

(i) If one has the estimation

$$
\mu\left(-A B^{-1}\right)+\frac{\|A\|\left\|B^{-1}\right\|^{2}\|C\|}{1-\left\|B^{-1}\right\|\|C\|}>0,
$$

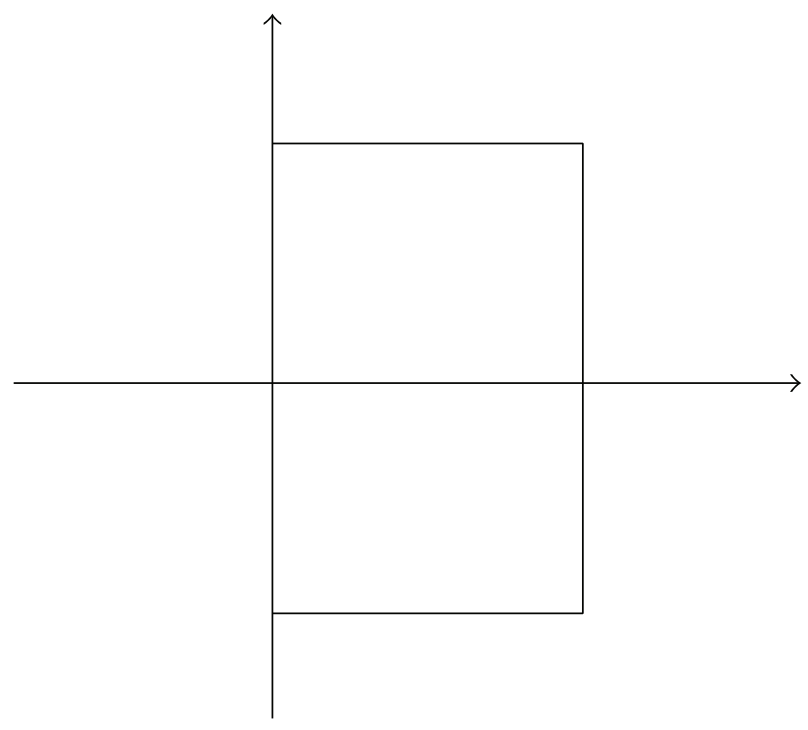

SCHEMe 1

then the inequalities

$$
\begin{gathered}
0 \leq \operatorname{Re} s \leq \mu\left(-A B^{-1}\right)+\frac{\|A\|\left\|B^{-1}\right\|^{2}\|C\|}{1-\left\|B^{-1}\right\|\|C\|}, \\
-\mu\left(-i A B^{-1}\right)-\frac{\|A\|\left\|B^{-1}\right\|^{2}\|C\|}{1-\left\|B^{-1}\right\|\|C\|} \leq \operatorname{Im}(s) \\
\leq \mu\left(i A B^{-1}\right)+\frac{\|A\|\left\|B^{-1}\right\|^{2}\|C\|}{1-\left\|B^{-1}\right\|\|C\|}
\end{gathered}
$$

hold (see Scheme 1).

(ii) If one has the estimation

$$
-\mu\left(A B^{-1}\right)-\frac{\|A\|\left\|B^{-1}\right\|^{2}\|C\|}{1-\left\|B^{-1}\right\|\|C\|}>0
$$

defining a positive number $\beta$ satisfying

$$
-\mu\left(A B^{-1}\right)-\frac{\|A\|\left\|B^{-1}\right\|^{2}\|C\|}{1-\left\|B^{-1}\right\|\|C\|} e^{-\beta \tau / m^{2}}=\beta,
$$

then the inequalities

$$
\begin{gathered}
\beta \leq \operatorname{Re} s \leq \mu\left(-A B^{-1}\right)+\frac{\|A\|\left\|B^{-1}\right\|^{2}\|C\|}{1-\left\|B^{-1}\right\|\|C\|} e^{-\beta \tau / m^{2}}, \\
-\mu\left(-i A B^{-1}\right)-\frac{\|A\|\left\|B^{-1}\right\|^{2}\|C\|}{1-\left\|B^{-1}\right\|\|C\|} e^{-\beta \tau / m^{2}} \leq \operatorname{Im} s \\
\quad \leq \mu\left(i A B^{-1}\right)+\frac{\|A\|\left\|B^{-1}\right\|^{2}\|C\|}{1-\left\|B^{-1}\right\|\|C\|} e^{-\beta \tau / m^{2}}
\end{gathered}
$$

are valid, where $m=\|A\|\left\|B^{-1}\right\|+\|A\|\left\|B^{-1}\right\|^{2}\|C\| /\left(1-\left\|B^{-1}\right\|\|C\|\right)$ (see Scheme 2). 


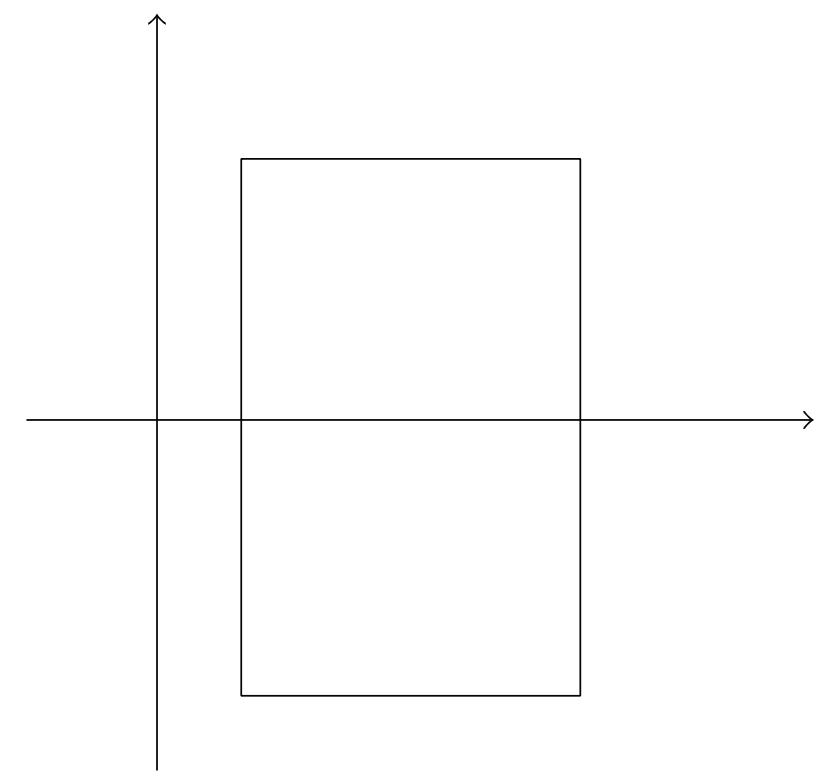

SCHEME 2

Proof. (i) A similar reduction to that of Lemma 8 yields

$$
0 \leq \operatorname{Re} s \leq \mu\left(-A B^{-1}\right)+\frac{\|A\|\left\|B^{-1}\right\|^{2}\|C\|}{1-\left\|B^{-1}\right\|\|C\|} .
$$

Noting that the imaginary part of an eigenvalue of a matrix $A$ is equal to the real part of an eigenvalue of $-i A$, we have the second inequality.

(ii) By Lemma 5,

$$
\begin{gathered}
-\mu\left[A\left(B+C e^{-\tau / s}\right)^{-1}\right] \leq \operatorname{Re} s \\
\leq \mu\left[-A\left(B+C e^{-\tau / s}\right)^{-1}\right] .
\end{gathered}
$$

It leads to

$$
\begin{gathered}
-\mu\left(A B^{-1}\right)-\frac{\|A\|\left\|B^{-1}\right\|^{2}\|C\|}{1-\left\|B^{-1}\right\|\|C\|} \leq \operatorname{Re} s \\
\leq \mu\left(-A B^{-1}\right)+\frac{\|A\|\left\|B^{-1}\right\|^{2}\|C\|}{1-\left\|B^{-1}\right\|\|C\|} .
\end{gathered}
$$

Let $\beta_{0}=-\mu\left(A B^{-1}\right)-\|A\|\left\|B^{-1}\right\|^{2}\|C\| /\left(1-\left\|B^{-1}\right\|\|C\|\right)$. Inequality (24) implies

$$
\begin{aligned}
-\operatorname{Re} s & \leq \mu\left[A\left(B+C e^{-\tau / s}\right)^{-1}\right] \\
& \leq \mu\left(A B^{-1}\right)+\frac{\|A\|\left\|B^{-1}\right\|^{2}\|C\|}{1-\left\|B^{-1}\right\|\|C\|}\left|e^{-\tau / s}\right| \\
& \leq \mu\left(A B^{-1}\right)+\frac{\|A\|\left\|B^{-1}\right\|^{2}\|C\|}{1-\left\|B^{-1}\right\|\|C\|} e^{-\operatorname{Re} s \cdot \tau / m^{2}},
\end{aligned}
$$

where the truth of the last inequality is attained from the following.

Set $s=s_{1}+s_{2} i$ and $s_{1}=\operatorname{Re} s>0$; then $\left|e^{-\tau / s}\right|=$ $\left|e^{-\tau /\left(s_{1}+s_{2} i\right)}\right|=e^{-s_{1} \tau /|s|^{2}}=e^{-\operatorname{Re} s \cdot \tau /|s|^{2}}$, where

$$
\begin{aligned}
|s| & \leq \rho\left[-A\left(B+C e^{-\tau / s}\right)^{-1}\right] \leq\left\|A\left(B+C e^{-\tau / s}\right)^{-1}\right\| \\
& =\left\|A\left(B^{-1}-B^{-1} C\left(I+B^{-1} C e^{-\tau / s}\right)^{-1} e^{-\tau / s} B^{-1}\right)\right\| \\
& \leq\|A\|\left\|B^{-1}\right\|+\frac{\|A\|\left\|B^{-1}\right\|^{2}\|C\|}{1-\left\|B^{-1}\right\|\|C\|}=m .
\end{aligned}
$$

So $\left|e^{-\tau / s}\right| \leq e^{-\operatorname{Re} s \cdot \tau / m^{2}}$, which means

$$
\operatorname{Re} s \geq-\mu\left(A B^{-1}\right)-\frac{\|A\|\left\|B^{-1}\right\|^{2}\|C\|}{1-\left\|B^{-1}\right\|\|C\|} e^{-\operatorname{Re} s \cdot \tau / m^{2}} .
$$

Hence, taking (25) into consideration, we have

$$
\operatorname{Re} s \geq-\mu\left(A B^{-1}\right)-\frac{\|A\|\left\|B^{-1}\right\|^{2}\|C\|}{1-\left\|B^{-1}\right\|\|C\|} e^{-\beta_{0} \cdot \tau / m^{2}} .
$$

Iteration

$$
\begin{aligned}
-\mu\left(A B^{-1}\right)-\frac{\|A\|\left\|B^{-1}\right\|^{2}\|C\|}{1-\left\|B^{-1}\right\|\|C\|} e^{-\beta_{j} \tau / m^{2}}= & \beta_{j+1} \\
& (j=0,1,2, \ldots)
\end{aligned}
$$

and the monotonicity

$$
\begin{gathered}
\beta_{0} \leq \beta_{1} \leq \cdots \leq \beta_{j} \leq \beta_{j+1} \leq \cdots \leq \operatorname{Re} s \\
\leq \mu\left(-A B^{-1}\right)+\frac{\|A\|\left\|B^{-1}\right\|^{2}\|C\|}{1-\left\|B^{-1}\right\|\|C\|}
\end{gathered}
$$

ensure that the limit of the series $\left\{\beta_{j}\right\}$ is equal to $\beta$, where $\beta$ is positive number satisfying

$$
-\mu\left(A B^{-1}\right)-\frac{\|A\|\left\|B^{-1}\right\|^{2}\|C\|}{1-\left\|B^{-1}\right\|\|C\|} e^{-\beta \tau / m^{2}}=\beta .
$$

Therefore, the first inequality holds. In a similar manner we can get the second inequality.

There is also another possible region including roots with nonnegative real parts which is illustrated in the following theorem.

Theorem 10. Let $\left\|B^{-1}\right\| \cdot\|C\|<1$. If s is a characteristic root of (12) with nonnegative real part, then the inequality

$$
|s| \leq \rho\left[|A| \cdot\left|B^{-1}\right| \cdot\left(I-\left|C B^{-1}\right|\right)^{-1}\right]
$$

holds. 


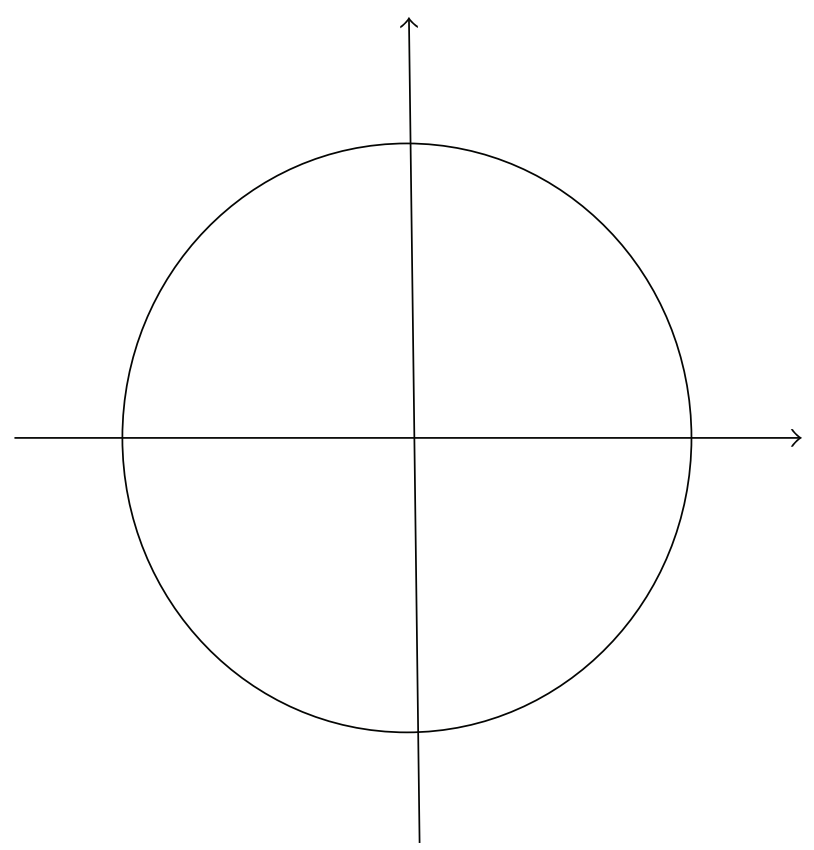

SCHEMe 3

Proof. By the assumption above, there exists an integer $j(1 \leq$ $j \leq d)$ such that

$$
s=\lambda_{j}\left[-A\left(B+C e^{-\tau / s}\right)^{-1}\right] .
$$

This implies the inequality

$$
|s| \leq \rho\left(-A\left(B+C e^{-\tau / s}\right)^{-1}\right) \text {. }
$$

It is obvious that

$$
\begin{aligned}
\left|-A\left(B+C e^{-\tau / s}\right)^{-1}\right| & =\left|A B^{-1}\left(I+C B^{-1} e^{-\tau / s}\right)^{-1}\right| \\
& \leq\left|A B^{-1}\right| \cdot \sum\left(I-\left|C B^{-1}\right|^{k}\right) \\
& \leq|A| \cdot\left|B^{-1}\right| \cdot\left(I-\left|C B^{-1}\right|\right)^{-1} .
\end{aligned}
$$

Therefore, due to Lemma 4, we have the conclusion.

In fact the region is a circle centered at the origin (see Scheme 3).

\section{Boundary Criteria for DDAEs}

Let

$$
\gamma=\mu\left(-A B^{-1}\right)+\frac{\|A\|\left\|B^{-1}\right\|^{2}\|C\|}{1-\left\|B^{-1}\right\|\|C\|} .
$$

By virtue of Lemma 8 , if $\gamma<0$, system (8) is asymptotically stable. If $\gamma \geq 0$, system (8) may be stable or unstable. We consider the stability of (8) when $\gamma \geq 0$.
Let $\beta_{0}=-\mu\left(A B^{-1}\right)-\|A\|\left\|B^{-1}\right\|^{2}\|C\| /\left(1-\left\|B^{-1}\right\|\|C\|\right)$ and $\gamma \geq 0$. We define the following quantities according to the sign of $\beta_{0}$ (see Theorem 9).

(i) If $\beta_{0} \leq 0$, then we put

$$
\begin{aligned}
& E_{0}=0, \\
& E=\mu\left(-A B^{-1}\right)+\frac{\|A\|\left\|B^{-1}\right\|^{2}\|C\|}{1-\left\|B^{-1}\right\|\|C\|}, \\
& F_{0}=-\mu\left(-i A B^{-1}\right)-\frac{\|A\|\left\|B^{-1}\right\|^{2}\|C\|}{1-\left\|B^{-1}\right\|\|C\|}, \\
& F=\mu\left(i A B^{-1}\right)+\frac{\|A\|\left\|B^{-1}\right\|^{2}\|C\|}{1-\left\|B^{-1}\right\|\|C\|} .
\end{aligned}
$$

(ii) If $\beta_{0}>0$, then we put

$$
\begin{aligned}
& E_{0}=\beta, \\
& E=\mu\left(-A B^{-1}\right)+\frac{\|A\|\left\|B^{-1}\right\|^{2}\|C\|}{1-\left\|B^{-1}\right\|\|C\|} e^{-\beta \tau / m^{2}}, \\
& F_{0}=-\mu\left(-i A B^{-1}\right)-\frac{\|A\|\left\|B^{-1}\right\|^{2}\|C\|}{1-\left\|B^{-1}\right\|\|C\|} e^{-\beta \tau / m^{2}}, \\
& F=\mu\left(i A B^{-1}\right)+\frac{\|A\|\left\|B^{-1}\right\|^{2}\|C\|}{1-\left\|B^{-1}\right\|\|C\|} e^{-\beta \tau / m^{2}},
\end{aligned}
$$

where $\beta$ is a root of the equation

$$
-\mu\left(A B^{-1}\right)-\frac{\|A\|\left\|B^{-1}\right\|^{2}\|C\|}{1-\left\|B^{-1}\right\|\|C\|} e^{-\beta \tau / m^{2}}=\beta .
$$

Under the above notations we turn our attention to the following three kinds of bounded regions in the $s$-plane.

Definition 11. Let $l_{1}, l_{2}, l_{3}$, and $l_{4}$ denote the segments $\left\{\left(E_{0}, y\right)\right.$ : $\left.F_{0}<y<F\right\},\left\{(x, F): E_{0} \leq x \leq E\right\},\left\{(E, y): F_{0} \leq y \leq F\right\}$, and $\left\{\left(x, F_{0}\right): E_{0} \leq x \leq E\right\}$, respectively. Furthermore, $l=$ $l_{1} \cup l_{2} \cup l_{3} \cup l_{4}$ and let $D$ be the rectangular region surrounded by $l$.

Definition 12. Let $R=\rho\left[|A| \cdot\left|B^{-1}\right| \cdot\left(I-\left|C B^{-1}\right|\right)^{-1}\right]$. Let $K$ denote the circular region with radius $R$ centered at the origin of the plane of $C$ :

$$
K=\{(r, \theta): r \leq R, 0 \leq \theta \leq 2 \pi\} .
$$

Definition 13. Let $T$ represent the intersection $D \cap K$. The boundary of $T$ is denoted by $\partial T$ and $\bar{T}=T \cup \partial T$.

A necessary and sufficient condition for the delayindependent stability of system (8) is presented in [6]. The following two theorems give criteria for the delay-dependent stability of system (8). We apply Theorems 1 and 2 to prove them, respectively. 


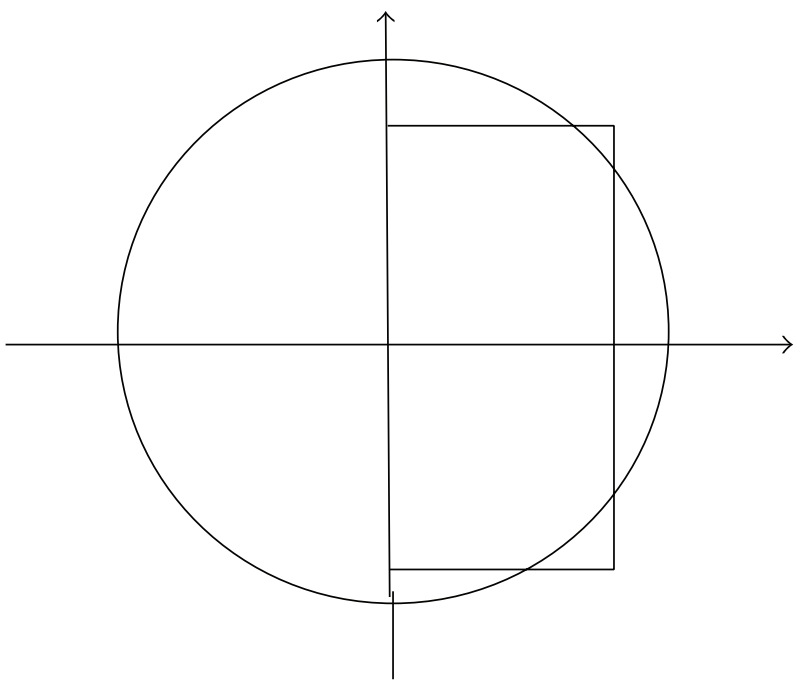

(a)

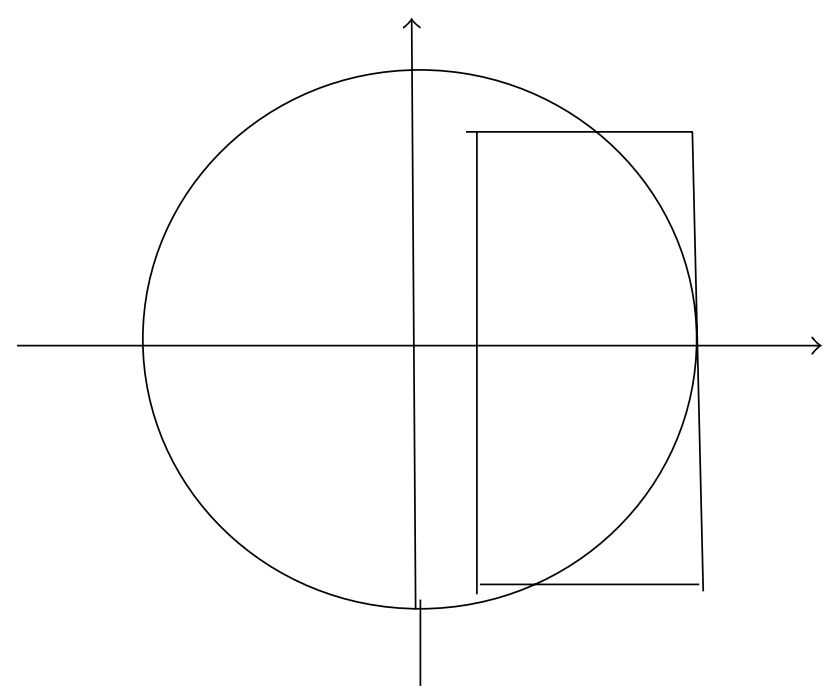

(b)

SCHEME 4

Theorem 14. If for any $(x, y) \in \partial T$, the real part $U(x, y)$ in (12) does not vanish, then system (8) is asymptotically stable.

Proof. Assume that the condition is satisfied and that system (8) is unstable, this means the existence of a characteristic root of (12) with nonnegative real part. According to Lemma 8, it suffices to prove $P(s) \neq 0$ for $\operatorname{Re} s \geq 0$. Applying Theorems 9 and 10 and Definition 13, it is sufficient to consider $s \in \bar{T}$. From the assumption of this theorem and statement of Theorem 9 , this contradicts with $P(s)=0$ for $s \in \bar{T}$. Hence, $P(s) \neq 0$ for $\operatorname{Re} s \geq 0$ and the proof is completed.

Due to Theorem 2, we can further extend the above result as follows.

Theorem 15. Assume that, for any $(x, y) \in \partial T$, there exists a real constant $\lambda$ satisfying

$$
U(x, y)+\lambda V(x, y) \neq 0
$$

Then system (8) is asymptotically stable.

The proof is analogous to Theorem 14. Two kinds of region $T$ considered in Definition 13 and Theorems 14 and 15 are as in Scheme 4.

\section{Conclusions}

We give two criteria for the delay-dependent stability of linear delay system (8). Theorems 9 and 10 show that the unstable characteristic roots of system (8) are located in some specified bounded region in the complex plane, while Theorems 14 and 15 show that it is sufficient to check certain conditions on its boundary to exclude the possibility of such roots from the region. Theorems 1 and 2 provide general and simple criteria for nonexistence of zeros of an analytic function in any boundary region.

\section{Conflict of Interests}

The authors declare that there is no conflict of interests regarding the publication of this paper.

\section{Acknowledgment}

The research is supported by Shanghai Natural Science Foundation (Grant no. 15ZR1431200).

\section{References}

[1] G.-D. Hu and G.-D. Hu, "Stability of discrete-delay systems: boundary criteria," Applied Mathematics and Computation, vol. 80, no. 2-3, pp. 95-104, 1996.

[2] C. A. Desoer and M. Vidyasagar, Feedback Systems: InputOutput Properties, Academic Press, New York, NY, USA, 1997.

[3] G. H. Golub and C. F. van Loan, Matrix Computations, Johns Hopkins Studies in the Mathematical Sciences, Johns Hopkins University Press, Baltimore, Md, USA, 3rd edition, 1996.

[4] W. Zhu and L. R. Petzold, "Asymptotic stability of linear delay differential-algebraic equations and numerical methods," Applied Numerical Mathematics, vol. 24, no. 2-3, pp. 247-264, 1997.

[5] J. X. Kuang and H. J. Tian, "The asymptotic behaviour of theoretical and numerical solutions for the differential equations with several delay terms," Journal of Shanghai Teachers University (Natural Sciences), vol. 23, pp. 1-9, 1994.

[6] Q. Lin and J. X. Kuang, "On the LD-stability of the nonlinear systems in MDBMs," Joural of Shanghai Teachers University (Natural Sciences), vol. 25, no. 4, 1996. 


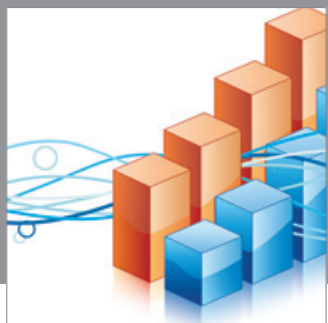

Advances in

Operations Research

mansans

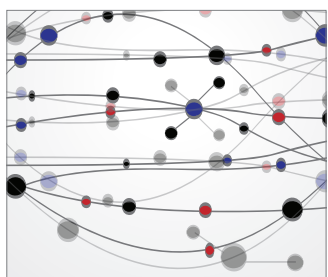

The Scientific World Journal
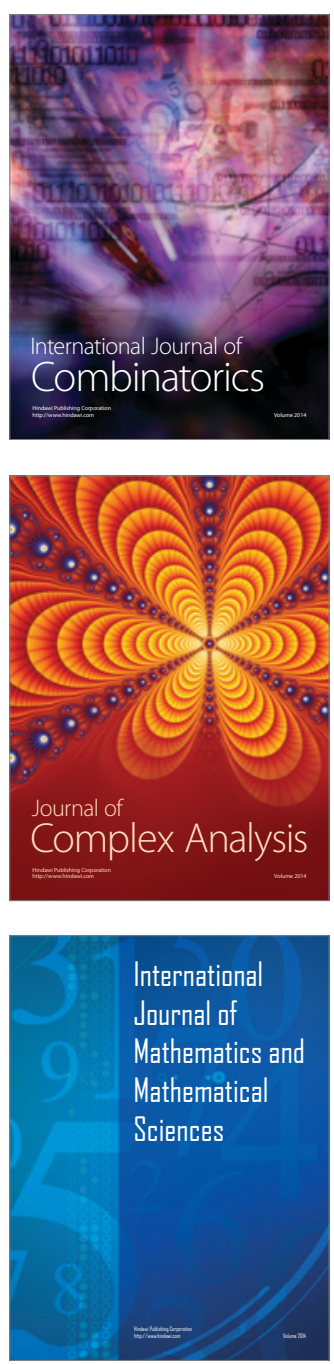
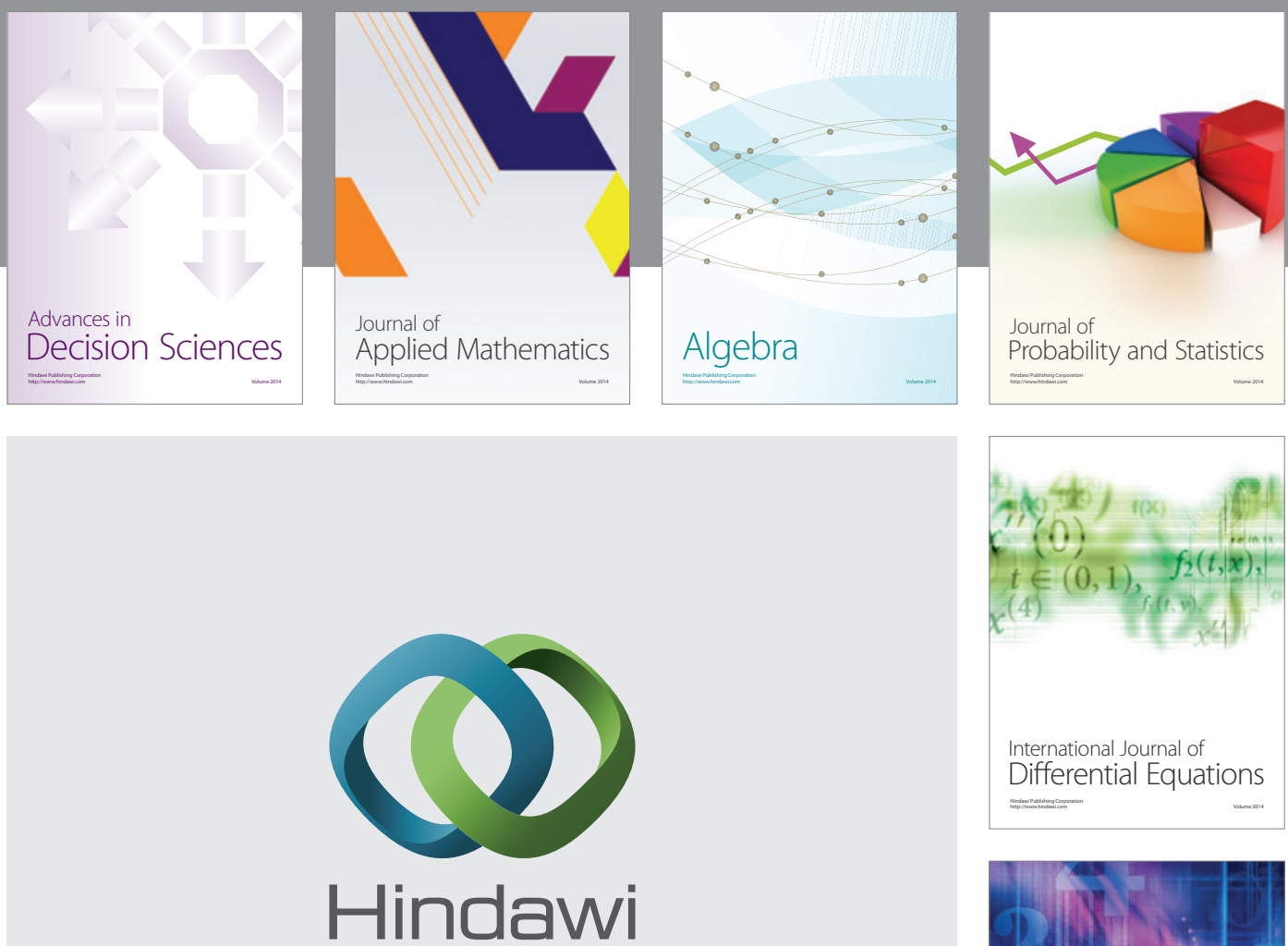

Submit your manuscripts at http://www.hindawi.com
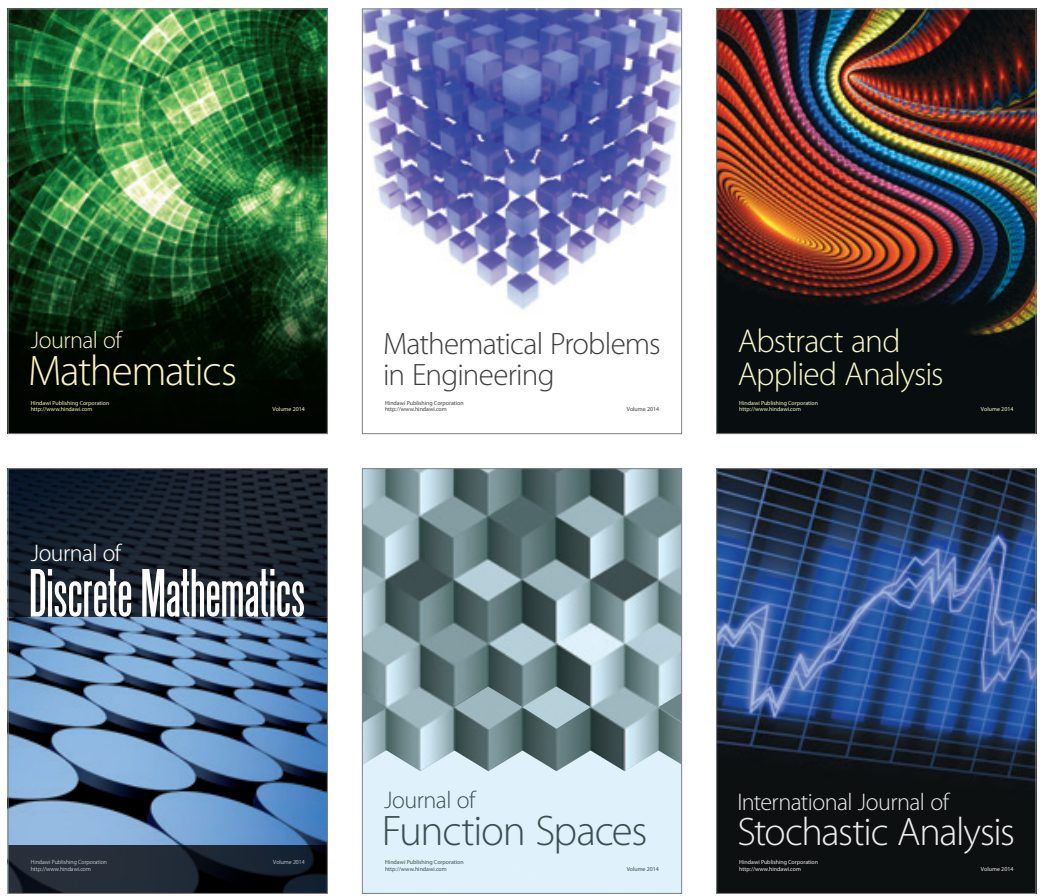

Journal of

Function Spaces

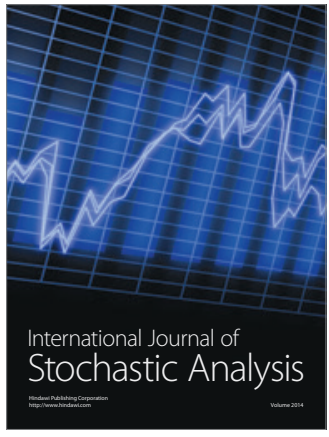

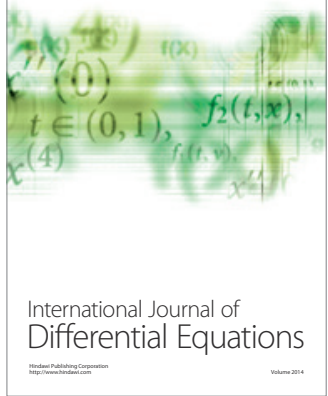
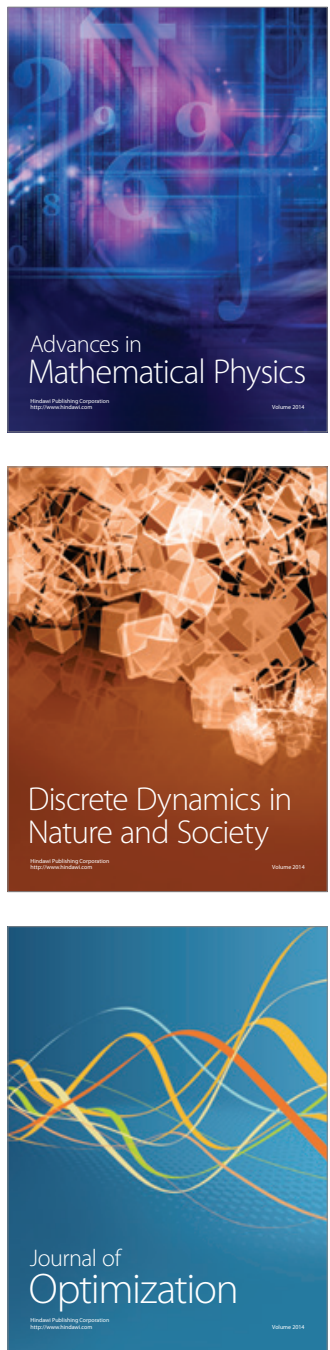